\title{
DETERMINAÇÃo DE ÁREA MÍNIMA PARA AQUISIÇÃo DE COLHEDORA PARA A COLHEITA DO ARROZ IRRIGADO NA FRONTEIRA OESTE DO RIO GRANDE DO SUL
}

\author{
Daniel Ciro de Souza ${ }^{*}$, Alexandre Russini ${ }^{2}$, Rogério Rodrigues de Vargas ${ }^{3}$, Eduardo Leonel Bottega ${ }^{4}$, José Fernando \\ Schlosser ${ }^{5}$ Marcelo Silveira Farias ${ }^{6}$ \\ 1,2 e 3 \\ Campus Itaqui, Universidade Federal do Pampa, 97650-000, Itaqui, Brasil. \\ ${ }^{4}$ Universidade Federal de Santa Maria, Campus de Cachoeira do Sul, 96503-205, Cachoeira do Sul, Brasil \\ ${ }^{5}$ Departamento de Engenharia Rural, Universidade Federal de Santa Maria, 97105-900, Santa Maria, Brasil. \\ ${ }^{6}$ Universidade Federal de Santa Maria, Campus de Frederico Westphalen, 98400-000, Frederico Westphalen, Brasil.
}

*E-mail: danielciro6@gmail.com

\section{RESUMO}

A colheita do arroz irrigado é considerada uma das operações mecanizadas mais importantes na cultura devido ao seu alto custo e valor agregado. Nesse sentido, o presente trabalho teve por objetivo realizar um estudo da área mínima de colheita que viabiliza a aquisição de uma colhedora automotriz nova para a cultura do arroz irrigado. $\mathrm{O}$ trabalho foi realizado no município de Itaqui, Fronteira Oeste do estado do Rio Grande do Sul (RS), a partir de dados de comercialização fornecidos pelos concessionários, sendo contempladas diferentes marcas e modelos de colhedoras, equipadas com sistemas de trilha do tipo axial, radial e híbrido. Para determinação da área mínima, foram analisados os custos fixos e variáveis, custo com colheita terceirizada, no ano agrícola de 2018/2019, e fator de redução de perdas por meio de equações matemáticas que consideram parâmetros relacionados às particularidades da cultura e da região. Os resultados definem que a menor área mínima que viabiliza a aquisição de uma colhedora automotriz nova para a cultura do arroz irrigado na região da Fronteira Oeste do estado é de 98,78 hectares. Portanto, quanto maior o nível tecnológico e as características dimensionais da colhedora, maior será a área mínima a ser colhida para justificar economicamente sua aquisição.

Palavras-chave: Oryza Sativa. Colheita Mecanizada. Viabilidade econômica.

\section{Introdução}

A cadeia orizícola desempenha papel importante cultural, social e economicamente no Brasil. $\mathrm{O}$ arroz é um dos cereais mais consumidos no país, sendo seu consumo anual estimado em 12 milhões de toneladas [1]. O estado do Rio Grande do Sul é o maior produtor de arroz, correspondendo a cerca de $45 \%$ da produção nacional [1]. A Fronteira Oeste é responsável por $35 \%$ da produção do estado, mesmo com a redução de 14,3\% na produção na safra de 2018/2019 [2]. Atualmente, todas as etapas que envolvem o processo produtivo são realizadas de forma mecanizada, que possibilita o aumento da produtividade, capacidade operacional de produção e área cultivada [3].

Nos sistemas de produção de arroz irrigado, a etapa que compete à colheita dos grãos é uma das mais importantes, sendo que isso ocorre devido ao elevado custo operacional em relação ao custo total de produção [4]. De acordo com Pacheco [5], a colheita corresponde a $11 \%$ dos gastos totais despendidos na produção do arroz irrigado, sendo que as máquinas podem representar entre 20 a $40 \%$ dos custos totais, devendo o produtor rural mensurar por meio do planejamento e dimensionamento do parque de máquinas se sua área comporta a aquisição de outra colhedora nova.

Quando se realiza o cálculo do custo de produção de grãos, as despesas decorrentes da atividade agrícola devem ser consideradas, o que leva a necessidade de uma administração apurada de toda a propriedade, visto que uma falha no planejamento poderá apresentar um impacto financeiro elevado no final da safra [6].

As particularidades da lavoura de arroz irrigado fazem com que a colheita se concentre em um período estreito de 
execução, com jornadas diárias reduzidas devido à umidade excessiva, bem como às baixas velocidades de trabalho. Martins et al. [7] destacam que ao ter um período menor para realizar a colheita, os produtores acabam utilizando número maior de colhedoras, obrigando-os a adquirirem máquinas novas e/ou terceirizar a operação. A aquisição de máquinas, visando atender uma demanda momentânea, é um risco, em função da ociosidade em anos normais, o que eleva de forma considerável o custo operacional, podendo, inclusive, inviabilizar a permanência da máquina na propriedade.

Com base no exposto, este trabalho teve por objetivo determinar a área mínima de colheita que viabiliza a aquisição de uma colhedora automotriz nova para colheita da cultura do arroz irrigado na região da Fronteira Oeste do estado do Rio Grande do Sul.

\section{Metodologia}

A pesquisa foi realizada na Universidade Federal do Pampa (UNIPAMPA), Campus Itaqui, Latitude 2907'31" S, Longitude 56³3'11" W, localizada na Fronteira Oeste do estado do Rio Grande do Sul. Foram utilizados dados referentes aos valores de venda das colhedoras novas e recebimento das usadas, considerando-se os modelos de colhedoras de maior comercialização no ano agrícola 2018/2019, bem como os custos com manutenção fornecidos pelos concessionários da região. Essas informações contemplam colhedoras equipadas com sistema de trilha axial, radial e híbrido, com plataformas do tipo draper e helicoidal, com potências nominais do motor na faixa de $200 \mathrm{cv}(147 \mathrm{~kW})$ a $448 \mathrm{cv}(330 \mathrm{~kW})$. As especificações técnicas das colhedoras utilizadas no estudo são apresentadas no Quadro 1.

Para estimativa da área mínima foi utilizada a metodologia proposta por Bottega et al. [6], os quais consideram, por meio de equações, os custos fixos e variáveis, valor da colheita terceirizada, bem como o fator de redução de perdas de colheita.

Os valores das larguras efetivas das plataformas das colhedoras de arroz e a velocidade de trabalho recomendada para colheita da cultura foram utilizadas para determinação da capacidade operacional da máquina, ou seja, da área colhida em função da unidade de tempo $\left(\right.$ ha $\left.\mathrm{h}^{-1}\right)$.

Com relação aos preços médios de comercialização do arroz na Fronteira Oeste do Rio Grande do Sul, entre os anos de 2018 a 2019, tomou-se como base o valor de $\mathrm{R} \$ 39,00$ a saca de $50 \mathrm{~kg}$ [2]. A produtividade média adotada nos cálculos foi de $8.100 \mathrm{~kg} \mathrm{ha}^{-1}$, valor superior à produtividade média da região (7.669 $\mathrm{kg} \mathrm{ha}^{-1}$ ) [2], por considerar os produtores rurais que possuem elevado nível de tecnologia e que adquirem as marcas e modelos de máquinas abordadas neste estudo.
Para estimar os custos fixos anuais das colhedoras, foi utilizado o método proposto por Debertolis et al. [8] e Bottega et al. [6], sendo considerados os custos com a depreciação anual, manutenção, juros e seguro, conforme a vida útil da máquina.

Foi utilizado para determinação do valor de depreciação o método linear (Equação 1). Nesse método, a linha de depreciação é constante ao ano, resultando na depreciação constante anual durante a vida útil da máquina [9].

$$
D=\frac{(V n-V s)}{V u a}
$$

Onde:

$\mathrm{D}=$ Depreciação $\left(\mathrm{R} \$ \mathrm{ano}^{-1}\right)$;

$\mathrm{Vn}=$ Valor de aquisição da colhedora nova $(\mathrm{R} \$)$;

$\mathrm{Vs}=$ Valor de sucata da colhedora $(\mathrm{R} \$)$;

Vua $=$ Vida útil (ano).

O valor de sucata foi considerado como valor de revenda, sendo que em função das particularidades da cultura do arroz irrigado, na qual promove desgaste excessivo dos principais componentes da máquina, o valor médio de revenda utilizado na região é de $40 \%$ em relação ao valor da colhedora nova. A vida útil do bem, ou seja, o espaço de tempo entre a compra e a rejeição de uma máquina ou implemento agrícola, seja esta rejeição por obsolescência ou por desgaste natural, foi considerado de 10 anos, conforme proposto por Da Silva et al. [10]. O custo com manutenção foi estimado conforme proposto por Debertolis et al. [8] e Keleski et al. [11], que remontam a uma taxa de manutenção equivalente a $70 \%$ do valor da colhedora nova para toda sua vida útil (Equação 2):

$$
C m=\frac{(V n \cdot T m)}{V u a}
$$

Onde:

$\mathrm{Cm}=$ Custo com manutenção $\left(\mathrm{R} \$ \mathrm{ano}^{-1}\right)$;

$\mathrm{Vn}=$ Valor do novo em moeda corrente $(\mathrm{R} \$)$;

Tm = Taxa de manutenção anual (0,70 a.a.);

Vua $=$ Vida útil (ano).

Quanto aos custos referentes às taxas de juros e de seguro foram realizados conforme as Equações 3 e 4, respectivamente:

$$
J=\frac{(V n+V s)}{2} \cdot i
$$

Onde:

$\mathrm{J}=\mathrm{Juros}\left(\mathrm{R} \$ \mathrm{ano}^{-1}\right)$;

$\mathrm{i}=$ Taxa anual de juros praticada pelo BNDES (2,5\% a.a). 
Quadro 1: Especificações técnicas, velocidade de deslocamento e valor de mercado das colhedoras utilizadas nas estimativas.

\begin{tabular}{|c|c|c|c|c|c|c|}
\hline Fabricante & Modelo & Pot. $(\mathrm{cv})^{1}$ & $\mathrm{ST}^{2}$ & L. $(\mathrm{m})^{3}$ & Vel. $\left(\mathrm{km} \mathrm{h}^{-1}\right)^{4}$ & $\operatorname{Vn}(R \$)^{5}$ \\
\hline (A) & a1 & 370 & Axial & 9,144 & 2,4 & $1.100 .000,00$ \\
\hline (A) & a2 & 305 & Axial & 9,144 & 2,2 & $880.000,00$ \\
\hline (B) & $\mathrm{b} 2$ & 448 & Axial & 10,668 & 2,2 & $1.280 .000,00$ \\
\hline (C) & $\mathrm{c} 1$ & 200 & Axial & 6,096 & 2,0 & $500.000,00$ \\
\hline (D) & $\mathrm{d} 1$ & 225 & Híbrida & 6,096 & 2,0 & $520.000,00$ \\
\hline (D) & $\mathrm{d} 2$ & 265 & Axial & 7,62 & 2,0 & $550.000,00$ \\
\hline$(\mathrm{E})$ & $\mathrm{e} 1$ & 223 & Radial & 6,096 & 2,0 & $450.000,00$ \\
\hline (E) & $\mathrm{e} 2$ & 280 & Híbrida & 7,62 & 2,2 & $890.000,00$ \\
\hline
\end{tabular}

${ }^{1}$ Potência máxima do motor; ${ }^{2}$ Sistema de trilha ${ }^{3}$ Largura da plataforma de corte; ${ }^{4}$ Velocidade de trabalho; ${ }^{5}$ Valor do bem novo, cotado junto ao concessionário.

Onde:

$$
S=\frac{(V n+V s)}{2} \cdot T s
$$

$\mathrm{S}=$ Seguro $\left(\mathrm{R} \$ \mathrm{ano}^{-1}\right)$;

Ts = Taxa anual de seguros (1,2\% a.a.).

O custo fixo anual (Cfa) foi obtido pela somatória de: depreciação (D), custo com manutenção $(\mathrm{Cm})$, juros $(\mathrm{J})$ e seguro (S), conforme a equação (5):

$$
C f a=D+C m+J+S
$$

Com a estimativa do custo fixo e o valor referente ao fator de redução de perdas de colheita compreendendo a porcentagem obtida a partir da razão entre a produtividade e o preço por saca, determinou-se o custo variável de cada colhedora, incluindo o valor que seria gasto com a colheita terceirizada. $\mathrm{Na}$ estimativa, ainda foram considerados os custos com combustível, lubrificantes e mão de obra.

Para o cálculo do fator de redução de perdas referentes à colheita com colhedora própria foi considerada a taxa de $2 \%$ a.a., proposta por Roessing [12] também utilizado por Bottega et al. [6], para colhedoras com sistema de trilha de fluxo radial. Já para máquinas com sistema de trilha de fluxo axial, foi considerada uma taxa de $1 \%$ a.a.

Para a determinação do consumo de combustível das colhedoras foi utilizado o método proposto por Piacentini [13] e [6], no qual o cálculo refere-se a razão entre a capacidade efetiva de colheita (ha $\mathrm{h}^{-1}$ ) e o consumo horário de combustível, multiplicado pelo custo do litro de combustível $(\mathrm{R} \$)$. Esse método proposto por Piacentini [13] estima o consumo horário de combustível em função da potência do motor, considerando um consumo de 0,2135 $\mathrm{L} \mathrm{kWh}^{-1}$ (Equação 6).

$$
C h=P .0,7355 \cdot 0,2135
$$

Onde:

$\mathrm{Ch}=$ Consumo horário de combustível $\left(\mathrm{L} \mathrm{h}^{-1}\right)$;

$\mathrm{P}=$ Potência do motor (cv);

$0,7355=$ Constante de conversão de potência $(\mathrm{cv}$ para $\mathrm{kW})$;

$0,2135=$ Constante de consumo.

Depois de obter o consumo horário de combustível de cada colhedora para estimativa do custo com combustível por unidade de área colhida, foi determinado o valor da capacidade efetiva de colheita de cada máquina. Para isso, utilizou-se a estimativa da capacidade efetiva de colheita proposta pela ASAE 496.2 [14] (Equação 7), considerando uma eficiência de colheita de 65\%, prevista pela ASAE 497.6 [15]; bem como por ARALDI et.al [16], em função das especificidades da cultura, na qual é exigida um maior número de paradas para descarga e tempo gasto com manobras.

$$
C T e=\frac{L . V}{10} \cdot f
$$

Onde:

$\mathrm{CTe}=$ Capacidade efetiva de colheita $\left(\mathrm{ha}^{-1}\right)$;

$\mathrm{L}=$ Largura da plataforma de corte $(\mathrm{m})$;

$\mathrm{V}=$ Velocidade de trabalho $\left(\mathrm{km} \mathrm{h}^{-1}\right)$;

$\mathrm{f}=$ Eficiência de campo (adimensional).

A partir da estimativa da capacidade efetiva de colheita, foi determinado o custo com combustível por unidade de área 
colhida (Equação 8), considerando-se o valor de $\mathrm{R} \$ 3,299$ para o litro de óleo diesel.

$$
C c=\frac{C h}{C T e} \cdot C l
$$

Onde:

$\mathrm{Cc}=$ Custo com combustível $\left(\mathrm{R} \$\right.$ ha $\left.^{-1}\right)$;

$\mathrm{Ch}=$ Consumo horário de combustível $\left(\mathrm{L} \mathrm{h}^{-1}\right)$;

$\mathrm{CTe}=$ Capacidade efetiva de colheita $\left(\mathrm{ha} \mathrm{h}^{-1}\right)$;

$\mathrm{Cl}=$ Custo do litro de combustível $(\mathrm{R} \$)$.

Referente aos custos com lubrificantes, considerou-se $15 \%$ do valor do custo com combustível, conforme proposto pela ASAE 496.2 [14]. Para a colheita terceirizada (Cct), cobra-se na região, em média, $8 \%$ da produção obtida, sendo que o custo referente à mão de obra $(\mathrm{Cmo})$, foi obtido considerando 1,5 salários mínimo pagos por mês no ano de 2019, conforme definido pelo Sindicato dos Trabalhadores Rurais, incluindo os encargos sociais em 12 meses. Não foram consideradas bonificações referentes à produção.

O custo variável foi estimado pela soma dos custos combustível, do litro de combustível e com mão de obra, conforme a Equação (9).

$$
C V=C c+C l+C m o
$$

Onde:

$\mathrm{CV}=$ Custo variável $\left(\mathrm{R} \$ \mathrm{ha}^{-1}\right)$;

$\mathrm{Cc}=$ Custo com combustível $\left(\mathrm{R} \$ \mathrm{ha}^{-1}\right)$;

$\mathrm{Cl}=$ Custo do litro de combustível $(\mathrm{R} \$)$;

$\mathrm{Cmo}=$ Custo com mão de obra $(\mathrm{R} \$)$.

A estimativa da área mínima cultivada de arroz irrigado (Equação 10), na qual viabiliza a aquisição de uma colhedora automotriz nova, foi realizada conforme o método proposto por Guimarães [17] e [6]; São considerados o custo fixo anual, o custo da colheita terceirizada, a redução de perdas de colheita e o custo variável por hectare.

$$
A m=\frac{C f a}{(C c t+R p)-C V}
$$

Onde:

Am = Área mínima a ser cultivada (ha);

$\mathrm{Cfa}=$ Custo fixo anual $(\mathrm{R} \$)$;

$\mathrm{Cct}=$ Custo da colheita terceirizada $\left(\mathrm{R} \$ \mathrm{ha}^{-1}\right)$;

$\mathrm{Rp}=$ Redução de perdas de colheita $(\mathrm{R} \$)$;

$\mathrm{CV}=$ Custo variável $\left(\mathrm{R} \$ \mathrm{ha}^{-1}\right)$.

\section{Resultados e discussões}

Os resultados referentes ao valor de revenda para cada colhedora avaliada no estudo são apresentados na Tabela 1.

Tabela 1: Valor de revenda das colhedoras automotrizes utilizadas no estudo.

\begin{tabular}{ccc}
\hline Marca & Modelo & Vs $(\mathbf{R} \$)^{\mathbf{1}}$ \\
\hline (A) & a1 & $440.000,00$ \\
(A) & a2 & $352.000,00$ \\
(B) & b1 & $460.000,00$ \\
(B) & b2 & $512.000,00$ \\
(C) & c1 & $200.000,00$ \\
(C) & c2 & $400.000,00$ \\
(D) & d1 & $208.000,00$ \\
(D) & d2 & $220.000,00$ \\
(E) & e1 & $180.000,00$ \\
(E) & e2 & $356.000,00$ \\
\hline
\end{tabular}

${ }^{1}$ Valor de sucata é considerado o valor de revenda do concessionário (40\%).

As colhedoras que possuem elevado valor de aquisição são as que apresentam os maiores valores de depreciação e manutenção. Esse comportamento se explica devido à complexidade de sistemas e do nível de tecnologia embarcada, que elevam o preço inicial em relação as outras marcas e modelos, e por exigirem manutenção qualificada. Corroborando com o resultado exposto, Hoffmann et al. [18] afirmam que a depreciação é inerente ao custo referente ao bem, decorrente do desgaste (depreciação física) e devido às inovações técnicas que ocorrem ao longo dos anos (obsolescência). Ainda, Garcia \& Silva [19] definem que o custo referente à depreciação deve ser debitado independente da máquina ser utilizada ou não.

Os resultados referentes ao consumo horário de combustível e os custos com lubrificantes e combustível das colhedoras estudadas são apresentados na Tabela 2.

\begin{tabular}{|c|c|c|c|c|c|}
\hline Colhedora & Modelo & $\begin{array}{l}\text { Pot } \\
\text { (cv) }\end{array}$ & $\begin{array}{c}\text { Ch } \\
\left(\mathbf{L ~ h} \mathbf{h}^{-1}\right) \\
\end{array}$ & $\begin{array}{c}\text { Cc } \\
\left(\mathbf{R} \$ \text { ha }^{-1}\right)\end{array}$ & $\begin{array}{c}\text { Club } \\
\left(\mathrm{R} \$ \mathrm{ha}^{-1}\right)^{1}\end{array}$ \\
\hline (A) & a1 & 370 & 58,10 & 134,43 & 154,29 \\
\hline (A) & $\mathrm{a} 2$ & 305 & 47,89 & 120,89 & 139,02 \\
\hline (B) & b1 & 326 & 51,19 & 118,44 & 136,21 \\
\hline (B) & b2 & 448 & 70,35 & 152,13 & 174,94 \\
\hline (C) & $\mathrm{c} 1$ & 200 & 31,41 & 130,74 & 150,35 \\
\hline (C) & $\mathrm{c} 2$ & 370 & 58,10 & 146,59 & 168,57 \\
\hline (D) & d1 & 225 & 35,36 & 147,08 & 169,14 \\
\hline (D) & $\mathrm{d} 2$ & 265 & 41,61 & 138,58 & 159,37 \\
\hline (E) & e1 & 223 & 35,02 & 145,77 & 167,63 \\
\hline (E) & $\mathrm{e} 2$ & 270 & 43,97 & 133,12 & 153,09 \\
\hline
\end{tabular}

Tabela 2: Consumo horário de combustível (Ch), custo com combustível (Cc) e custo com lubrificante (Club) das colhedoras automotrizes utilizadas no estudo.

${ }^{1}$ Custo com lubrificante: $15 \%$ do custo com combustível

O maior consumo foi observado para a colhedora (B), modelo b2, sendo que o menor corresponde a colhedora (C), modelo c1. No entanto, o consumo horário de máquinas agrícolas é de difícil mensuração devido haver diversas variáveis de cálculo que devem ser consideradas como, por exemplo, a habilidade do 
operador na manobra da colhedora, o tipo de terreno relacionado às características do solo e sua topografia [6].

Na Tabela 3, são apresentados os valores dos Custos com depreciação (D), manutenção (M), juros (J) e seguro (S), estimados anualmente para todas as colhedoras estudadas.

Tabela 3: Custos com: depreciação (D); manutenção (M); juros (J) e seguro (S), estimados anualmente para as colhedoras estudadas.

\begin{tabular}{|c|c|c|c|c|c|}
\hline Colhedora & Modelo & $\begin{array}{l}\text { D (R\$ } \\
\left.\text { ano-1 }^{-1}\right)\end{array}$ & $\begin{array}{l}\text { M }(\mathrm{R} \$ \\
\left.\mathrm{ano}^{-1}\right)^{1}\end{array}$ & $\begin{array}{c}\mathrm{J}(\mathbf{R} \$ \\
\left.\mathrm{ano}^{-1}\right)^{2}\end{array}$ & $\begin{array}{c}\mathrm{S}(\mathrm{R} \$ \\
\left.\mathrm{ano}^{-1}\right)^{3}\end{array}$ \\
\hline (A) & a1 & $66.000,00$ & $77.000,00$ & $19.250,00$ & $9.240,00$ \\
\hline (A) & $\mathrm{a} 2$ & $52.800,00$ & $61.600,00$ & $15.400,00$ & $7.392,00$ \\
\hline (B) & b1 & $69.000,00$ & $80.500,00$ & $20.125,00$ & $9.660,00$ \\
\hline (B) & b2 & $76.800,00$ & $89.600,00$ & $22.400,00$ & $10.752,00$ \\
\hline (C) & $\mathrm{c} 1$ & $30.000,00$ & $35.000,00$ & $8.750,00$ & $4.200,00$ \\
\hline (C) & $\mathrm{c} 2$ & $60.000,00$ & $70.000,00$ & $17.500,00$ & $8.400,00$ \\
\hline (D) & d1 & $31.200,00$ & $36.400,00$ & $9.100,00$ & $4.368,00$ \\
\hline (D) & $\mathrm{d} 2$ & $33.000,00$ & $38.500,00$ & $9.625,00$ & $4.620,00$ \\
\hline (E) & e1 & $27.000,00$ & $31.500,00$ & $7.875,00$ & $3.780,00$ \\
\hline (E) & e2 & $53.400,00$ & $62.300,00$ & $15.575,00$ & $7.476,00$ \\
\hline
\end{tabular}

${ }^{1}$ Taxa de manutenção considerada no estudo: $70 \%$ do valor do bem novo. ${ }^{2}$ Taxa de juros: 2,5\% a.a. (média do rendimento real anual da poupança nos últimos 10 anos. FONTE: Banco Central. Dados de Agosto de 2018). ${ }^{3}$ Taxa de seguro: $1,2 \%$

Ressalta-se que, máquinas com maior nível de componentes eletrônicos sofrem maior depreciação devido à obsolescência, fator este que deve ser muito bem observado no momento da aquisição de uma colhedora nova. Esse comportamento vai ao encontro do proposto por Consetino [9], o qual descreve que a depreciação de uma máquina agrícola ocorre independente de sua utilização, sendo considerado o maior custo de uma máquina agrícola. Ainda, conforme o autor, é importante sua análise, pois determinará quando a máquina deve ou não ser substituída, devido à sua capacidade produtiva obsoleta ou quando se torna obsoleta em função do surgimento de novas tecnologias.

Quanto ao custo de manutenção, as colhedoras que apresentam elevada capacidade operacional são submetidas à intensa utilização devido ao seu elevado custo final, a fỉm de justificar sua aquisição. Logo, poderão apresentar desgaste excessivo de seus componentes, principalmente, na cultura do arroz irrigado, devido à sua abrasividade, exigindo cuidados adicionais com manutenção [7].

Referente ao custo fixo (Tabela 4), o maior valor observado recai sobre a colhedora (B), modelo b2 e o menor para a (E), modelo e1, diferença de 64,84\%. Quanto ao custo variável, o maior valor foi referente à colhedora (D), modelo d1 sendo o menor para a colhedora (B), modelo b1, correspondendo a uma diferença de $21,42 \%$ entre as colhedoras analisadas.
Tabela 4: Estimativas do custo fixo anual $\left(\mathrm{R} \$ \mathrm{ano}^{-1}\right)$ e do custo variável $\left(\mathrm{R} \$ \mathrm{ha}^{-1}\right)$, para as colhedoras estudadas.

\begin{tabular}{cccc}
\hline Colhedora & Modelo & Cfa $\left(\mathbf{R} \$ \mathbf{~ a n o}^{-1}\right)$ & Cv $\left(\mathbf{R} \$ \mathbf{~ h a}^{-\mathbf{1}}\right)$ \\
\hline (A) & a1 & $171.490,00$ & 146,35 \\
(A) & a2 & $137.192,00$ & 133,60 \\
(B) & b1 & $179.285,00$ & 130,37 \\
(B) & b2 & $199.552,00$ & 163,49 \\
(C) & c1 & $77.950,00$ & 149,56 \\
(C) & c2 & $155.900,00$ & 159,29 \\
(D) & d1 & $81.068,00$ & 165,90 \\
(D) & d2 & $85.745,00$ & 154,30 \\
(E) & e1 & $70.155,00$ & 164,59 \\
(E) & e2 & $138.751,00$ & 147,70 \\
\hline
\end{tabular}

O custo fixo anual varia conforme os valores de aquisição de cada máquina nova, sendo que quanto maior o investimento, maior será o custo anual [6]. Os autores destacam ainda que os custos variáveis estão diretamente ligados a outros fatores como: velocidade de trabalho, rendimento operacional, largura da plataforma e tipo de sistema de trilha, justificando os resultados obtidos.Nesse sentido, para um mesmo intervalo de tempo, quanto maior for a largura da plataforma e mais elevada a velocidade de trabalho, maior será a área que a máquina irá colher, reduzindo o custo fixo por unidade de área colhida.

Na Tabela 5, são apresentadas as estimativas de área mínima a ser cultivada com arroz irrigado que viabiliza a aquisição de uma colhedora automotriz nova.

Tabela 5: Estimativa da área mínima (Am), para as colhedoras avaliadas.

\begin{tabular}{ccc}
\hline Colhedora & Modelo & Am (hectares) \\
\hline (A) & a1 & 235,42 \\
(A) & a2 & 185,09 \\
(B) & b1 & 240,83 \\
(B) & b2 & 280,54 \\
(C) & c1 & 107,48 \\
(C) & c2 & 217,89 \\
(D) & d1 & 114,36 \\
(D) & d2 & 119,01 \\
(E) & e1 & 98,78 \\
(E) & e2 & 190,83 \\
\hline
\end{tabular}

$\mathrm{Am}^{1}$ : Área mínima calculada considerando a redução de perdas de colheita.

Observa-se que a menor área mínima que viabiliza a aquisição de uma colhedora nova corresponde a 98,78 hectares, (colhedora marca E, modelo e1), sendo essa, a colhedora que apresentou o menor valor de aquisição. Porém, o maior valor de área mínima corresponde a 280,54 hectares, referente à colhedora da marca B, modelo b2, a qual, justamente apresenta o maior custo fixo anual.

Quando comparado às outras culturas como soja, milho e trigo pode-se inferir que a área mínima para a cultura do arroz irrigado na qual justifica a aquisição de uma colhedora nova é menor. Corroborando, Bottega et al. [6], definem que a área mínima que justifique a viabilidade de aquisição de uma colhedora 
nova remete a 304,52 hectares, para as culturas da soja e milho, na região de Farol, no estado do Paraná.

As diferenças na área mínima em relação às outras culturas se devem às particularidades do cultivo do arroz irrigado, que se caracteriza por ser uma cultura anual, sendo que a colheita se concentra em um período estreito, que varia de 40 a 60 dias na região da Fronteira Oeste, do estado do Rio Grande do Sul. Além disso, as características como, elevada produtividade de grãos por hectare e volume de massa a ser processada pelos mecanismos internos da máquina, torna necessário que as colhedoras se desloquem a baixa velocidade, o que diminui consideravelmente a capacidade operacional.

Cabe ressaltar que neste estudo as colhedoras são utilizadas unicamente na cultura do arroz irrigado, tendo como consequência reduzida utilização anual, com elevada imobilização de capital, o que aumenta consideravelmente os custos fixos. Corroborando com o exposto, Milan [20] descreve que o dimensionamento acima da necessidade certamente ocasionaria aumento dos custos finais de produção, pela baixa utilização das máquinas e, consequentemente, aumento dos custos fixos. Esse fator é determinante para a sustentabilidade da cadeia orizícola, pois permite ao agricultor decidir qual máquina melhor se adequa ao planejamento de sua propriedade, bem como refletir sobre a compra ou aluguel de uma colhedora.

\section{Conclusões}

A área mínima que viabiliza a aquisição de uma colhedora automotriz nova para a colheita da cultura do arroz irrigado na região da Fronteira Oeste do estado do Rio Grande do Sul é variável entre os modelos analisados, sendo que o menor e o maior valor de área mínima correspondem a 98,78 e 280,50 hectares, respectivamente.

Quanto maior o nível tecnológico e as características dimensionais da colhedora, maior será a área mínima a ser colhida, para justificar a viabilidade econômica da sua aquisição.

\section{DETERMINATION OF MINIMUM AREA FOR ACQUISITION OF A COMBINE FOR THE HARVESTING OF IRRIGATED RICE ON THE WEST BORDER OF RIO GRANDE DO SUL}

ABSTRACT: The irrigated rice harvest is considered one of the most important mechanized operations in the crop, due to its high cost and added value. In this sense, the present work aimed to carry out a study of the minimum harvest area that makes possible the acquisition of a new self-propelled combine for the of irrigated rice crop. The work was carried out in the Itaqui city, West Frontier of Rio Grande do Sul State (RS), based on marketing data provided by the concessionaires, considering different brands and models of combines, equipped with axial type trail systems, radial and hybrid. To determine the minimum area, fixed and variable costs, cost of outsourced harvesting, in the 2018/2019 agricultural year, and loss reduction factor were analyzed using mathematical equations, which consider parameters related to the particularities of the crop and the region. The results define that the smallest minimum area that makes possible the acquisition of a new selfpropelled combine for the irrigated rice in the Western Frontier region of the state is 98.78 hectares. Therefore, the higher the technological level and the dimensional characteristics of the combine, the larger the minimum area to be harvested to economically justify its acquisition.

Keywords: Oryza Sativa. Mechanized harvest. Economic viability.

\section{Referências}

[1] CONAB. Perspectiva para agropecuária: safra 2018/19. v.6. Brasília, 2018. 51p. Disponível em:

<https://www.conab.gov.br/images/arquivos/outros/Perspectivas-para-aagropecuaria-2018-19.pdf>. Acesso em: 10 de jan 2020.

[2] INSTITUTO RIOGRANDENSE DO ARROZ. Custo de produção média ponderado arroz irrigado Rio Grande do Sul safra 2018/19: Revisão. (IRGA) Disponível em: <https://irgaadmin.rs.gov.br/upload/arquivos/201905/24135707-custo-revisado-safra-201819.pdf>. Acesso em: 20 nov. 2019

[3] CONAB. Acompanhamento da safra brasileira de grãos. v. 5. Safra 2017/18, n.5. Quinto levantamento, fevereiro 2018. Brasília, 2018. Disponível em:< file:///C:/Users/danie/Downloads/Boletim_Graos_fevereiro_2018.pdf>. Acesso em: 10 de dez. 2019.

[4] CUNHA, G. B. da. Gestão de Custo de Uma Unidade de Produção Agrícola no Município de Capivari do Sul, RS. 2011. 49f. Trabalho de Conclusão de Curso (Monografia) Graduação Tecnológica em Planejamento e Gestão para desenvolvimento Rural. Universidade Federal do Rio Grande do Sul. Disponível em:<https://lume.ufrgs.br/bitstream/handle/10183/38165/000820173.pdf?sequenc $\mathrm{e}=1>$. Acesso em: 15 de dez. 2019.

[5] PACHECO, E. P. Seleção e custo operacional de máquinas agrícolas. Rio Branco: Embrapa Acre, 2000. 21p. (Embrapa Acre. Documentos, 58). Disponível em: <https://www.infoteca.cnptia.embrapa.br/handle/doc/495355>. Acesso em: 15 de out. 2019.

[6] BOTTEGA, E. L. et al. Aquisição de uma colhedora combinada de grãos: estudo de viabilidade para a região de farol, PR, no ano agrícola de 2012. Scientia Agraria Paranaensis, Vol. 14, n. 2, abr./jun., p. 82-88, 2015. DOI: 10.18188/19831471/sap.v14. n.2.p82-88.

[7] MARTINS, L. D. N. et al. Ajuda Importante. Revista Cultivar Máquinas, v. 181, p. 33-35, 2018.

[8] DeBerTOlis, A. J.; ALEXIUS, M. L.; DOSSA, D. Trabalhador na administração de 295 empresas agrossilvipastoris. $2^{\circ}$. Ed. Revisada Curitiba: SENAR-PR, 2005.

[9] CONSENTINO, R. M. A. Modelo empírico de depreciação para tratores agrícolas de rodas. Dissertação (Mestrado). Escola Superior de agricultura Luiz de Queiroz. Universidade de São Paulo. Piracicaba, 2004. Disponível 


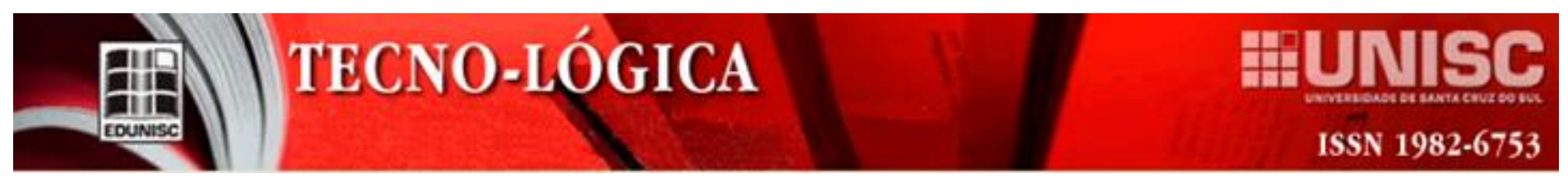

em:<https://www.teses.usp.br/teses/disponiveis/11/11148/tde-04032005-

162345/publico/rui.pdf >. Acesso em: 28 jun 2019.

[10] DA SILVA, R. P. et al. Custo horário de máquinas agrícolas. (Material didático) Universidade Estadual Paulista "Júlio De Mesquita Filho". Faculdade de Ciências Agrárias e Veterinárias Campus de Jaboticabal. Jaboticabal, 2015. Disponível

<http://www.fcav.unesp.br/Home/departamentos/engenhariarural/rouversonpereir adasilva/apostila-de-custo-de-operacoes-agricolas.pdf $>$. Acesso em: 28 de jun. 2019.

[11] KELESKI, A. et al. Análise da viabilidade econômica da cultura da soja na região de Campo Mourão/PR, safra 2013/14. Rev. Ciências Exatas e da Terra e Ciências Agrárias, Vol. 13, n. 1, p.09-18, jul./dez., 2018. Disponível $\mathrm{em}:\langle$ http://revista2.grupointegrado.br/revista/index.php/campodigital $>$. Acesso em: 28 Mar. 2019

[12] ROESSING, A. C. Tamanho ótimo de propriedade para aquisição de colhedeira de soja.Comunicado Técnico EMBRAPA, 1982. 7p. Disponível em:<https://ainfo.cnptia.embrapa.br/digital/bitstream/item/77516/1/CNPSO-

COM.-TEC.-14-82.pdf $>$. Acesso em: 28 de out. 2019.

[13]PIACENTINI, L. et al. Software para estimativa do custo operacional de maquinas agrícolas - Maqcontrol. Engenharia Agrícola Vol.32, n.3, p.609-623, 2012. DOI:https://doi.org/10.1590/S0100-69162012000300020.

[14] ASAE (AMERICAN SOCIETY OF AGRICULTURAL ENGINEERS). ASAE EP 496.2 FEB03: Agricultural machinery management data. In:

ASAE Standards 2003: standards engineering practices data. St. Joseph, 2003. p.366-372.ASAE (AMERICAN SOCIETY OF AGRICULTURAL ENGINEERS).

[15] ASAE EP 497.6 JUN09: Agricultural machinery management data. In: ASAE Standards 2009: standards engineering practices data. St. Joseph, 2009. p.350-357.

[16] ARALDI, P. F. et al. Eficiência operacional na colheita mecanizada em lavouras de arroz irrigado. Ciência Rural, Vol.43, n.3, p445-451, mar 2013. DOI: $10.1590 /$ S0103-84782013000300011

[17] GUIMARÃES, F. S. E. Máquinas para colheita. Cultivar Máquinas. Caderno técnico de máquinas, circular encartado na edição de julho/agosto de 2002. N.13, 10p. 2002.

[18]HOFFMANN, R. et al. Administração da empresa agrícola. 3. ed. São Paulo: Pioneira, 1987. $325 \mathrm{p}$.

[19]GARCIA, R. F \& SILVA, L. S. Avaliação do corte manual e mecanizado de cana-de-açúcar em Campos dos Goytacazes, RJ. Engenharia na Agricultura,

Viçosa - mg, Vol.18 n.3, p 234-240, mar-jun

2010.DOI: https://doi.org/10.13083/reveng.v18i3.67

[20] MILAN, M. Gestão sistêmica e planejamento de máquinas agrícolas. 2004. 100 p. Tese (Livre-Docência) - Escola Superior de Agricultura Luiz de Queiroz, Universidade de São Paulo, Piracicaba, 2004. 